\title{
An accurate instantaneous angular speed estimation method based on a dual detector setup
}

\author{
Qiang Zeng ${ }^{\mathrm{a}}$, Guojin Feng ${ }^{\mathrm{b}}$, Yimin Shao ${ }^{\mathrm{a}}$, James Devitt ${ }^{\mathrm{b}}$, Fengshou Gu ${ }^{\mathrm{b}}$, Andrew Ball ${ }^{\mathrm{b}}$ \\ a. State Key Laboratory of Mechanical Transmission, Chongqing University, Chongqing, PR China \\ ${ }^{\mathrm{b}}$ School of Computing and Engineering, University of Huddersfield, Huddersfield, UK
}

\begin{abstract}
Instantaneous angular speed (IAS), or 'true IAS', is an inherent signature directly related to the mechanical dynamics of rotor systems and has been employed extensively in different rotating machines. Due to various uncertainties, such as manufacturing error or the inaccurate installation of encoders, the acquired IAS signals often contains significant contents of 'false IAS' which can mask the small variations of IAS, lead to low accuracy and consequently affect the understanding of the dynamic behaviour of a rotor system. This study proposes a dual detector method to minimize the influence of encoder errors and hence obtain more accurate IAS estimation. Through theoretical study, it is proved that the false IAS induced by encoder errors from the two detectors have the same amplitudes but with a phase difference, whereas the true IAS components from the two detectors are identical. On this basis, an algorithm is developed firstly to acquire the encoder errors through a phase alignment operation and then estimate the true IAS after removing the estimated encoder errors. The proposed method is evaluated by both numerical simulations and experimental studies. The results show that the proposed method can more significantly suppress the encoder errors associated with encoder eccentricities in comparison with conventional reference methods, thereby yielding an accurate true IAS from the measurement. The proposed method can be particularly effective when the encoder has very low accuracy, allowing a more cost-effective IAS monitoring system to be achieved.
\end{abstract}

Key words: Instantaneous angular speed, Dual detector, Encoder error model, Encoder error suppression, Frequency domain demodulation

\section{Introduction}

Instantaneous angular speed (IAS) represents the transient angular speed variation and has been considered as an intrinsic signature to reflect rotor dynamics in a rotating mechanical system. It contains the rigid-body-motion part reflecting the machine operating condition and the torsional oscillation part reflecting the mechanical dynamics. Considering IAS can be acquired with an easy setup, it has been investigated extensively as an effective tool for the condition monitoring and fault diagnosis of rotating machineries. 
Literature shows IAS has been studied for a variety of common mechanical components such as the fixed-axis gearboxes [1], planetary gearbox [2][3], rolling bearings [4], engine-propellers [5], and rotor blades [6]. It can assist the implementation of order tracking under stationery and non-stationary conditions through an angular sampling operation [7][8]. The measured IAS contains the true IAS related to the mechanical dynamics together with the false IAS, which are generated by the factors like IAS estimation error, measurement parameter error or encoder errors, etc. In [9], measurement parameters, including time duration, sampling frequency and encoder resolution, were studied and it was concluded that longer time duration and/or a higher resolution encoder would result in lower background noises in the IAS spectrum. In [10], it is found that the ratio of the false IAS caused by encoders to the true one can be greater than as much as $60 \mathrm{~dB}$, indicating that the false IAS contains high energy and hence can be a strong noise source. The false IAS can mask weak IAS signatures and hence affect the understanding of true rotor behaviours, which can be particularly worse with the lowcost or the low-accuracy encoders. Ideally, the encoder would be perfect, with evenly distributed encoder division angles that generates a true IAS signal which then reveals the vital signatures of the rotor. However, in a real world application, the encoder is imperfect due to manufacturing or installation errors, as discussed in [11][12][13], often generating false IAS signals. Many studies have focused on this problem, aiming to suppress the encoder errors as well as the corresponding false IAS signals.

Existing methods suppressing encoder error can be catagorized into two groups: cross-correction methods and self-correction methods. Cross-correction methods require a reference device with better accuracy and resolution performance, such as a high accuracy turntable, a ring laser [14][15], an autocollimator with polyhedral [16], or an optical encoder [17], to calibrate the encoder and generate its specific error profile look-up-table (LUT). The drawbacks of cross-correction methods are obvious. They require a reference device to be installed on the same shaft of the target encoder and recalibration is needed once it is used on another shaft. To the authors' knowledge, cross-correction methods are usually applied in situations where high accuracy is necessary. Self-correction methods do not need a reference device, however, they usually require the angle intervals to be of high precision for multiple detector placements [18][19][20] or the rotor speed to change linearly [12][21]. The existing methods with multiple detectors are mainly based on prime-factor-division method (PFD)[18][19] and equaldivision-averaged method[20]. Despite corrected by the counting method or the elapsed time method, IAS estimation still faces the aliasing phenomenon due to discrete counting and has background noises across its whole frequency range due to quantification errors [22]. In addition, this method also requires a very high-frequency counter, typically of $80 \mathrm{MHz}$ to $100 \mathrm{MHz}$ [4][21], and generates tremendous data. In [23], Diamond et al. assume that the IAS is continuous and can be expressed as a second order polynomial between counting points, and the polynomial parameters can be solved with a Bayesian linear regression. This is a good method for low-resolution encoder and the estimation of a short-time IAS signal, however, the regression time increases rapidly with the increase of the input data length, which can reach nearly 90 min with 4740 counting points.

This paper proposes a novel method to accurately estimate IAS with a dual detector setup, in which 
the encoder errors can be directly suppressed, which is different from other methods in multiple detector setup that supress the encoder error by averaging or PFD. The theory of this method is that the true IAS is identically recorded by both detectors, while the false IAS from the two detectors has a phase shift which is dependent on the angle between the two detectors. The proposed method is an improvement on the frequency domain demodulation (FDD) method introduced in [9].

The rest of the paper is structured as follows. In Section 2, the conventional way to estimate IAS signal and encoder errors are firstly explained and then a new algorithm to estimate IAS signal with a dual detector setup is proposed. In Section 3, numerical simulations are conducted to evaluate the performance of the proposed method to suppress encoder errors and also the influence by factors, like the angle between the dual detector and the shaft rotating speed. Then, a test rig is established in Section 4 and runs at a quasi-constant speed condition to evaluate the proposed method by comparing with other well-accepted methods. Finally, conclusions are drawn in Section 5.

\section{Theoretical background}

This section firstly describes the conventional setup to acquire shaft rotating information and the common methods to estimate IAS signal. Then, the encoder errors that can affect the estimation of IAS signal are explained and the methods to supress such errors are also introduced. On this basis, a method to estimate IAS based on a two detector setup is proposed and an algorithm with two key steps to estimate the IAS signal is introduced.

\subsection{Conventional IAS estimation methods}

A typical setup to measure IAS signal is illustrated in Fig. 1(a), which includes an encoder disc and an optical detector. The encoder disc contains evenly divided slots to block/pass the light beam path. The optical detector includes an emitter to generate a light beam, and a receiver to receive the light beam and transform the received light into electric signal. In the setup, the encoder disc is fixed on and rotates together with the shaft, and the pair of optical emitter and receiver is placed oppositely on the two sides of the encoder disc. When the shaft rotates, the encoder disc successively block/pass the light beam from the emitter, resulting a pulse train signal can then be recorded by the receiver, as presented in Fig. 1 (b). By processing the pulse train signals, the rotating speed of the shaft, i.e. IAS signal, can then be estimated.

(a) 

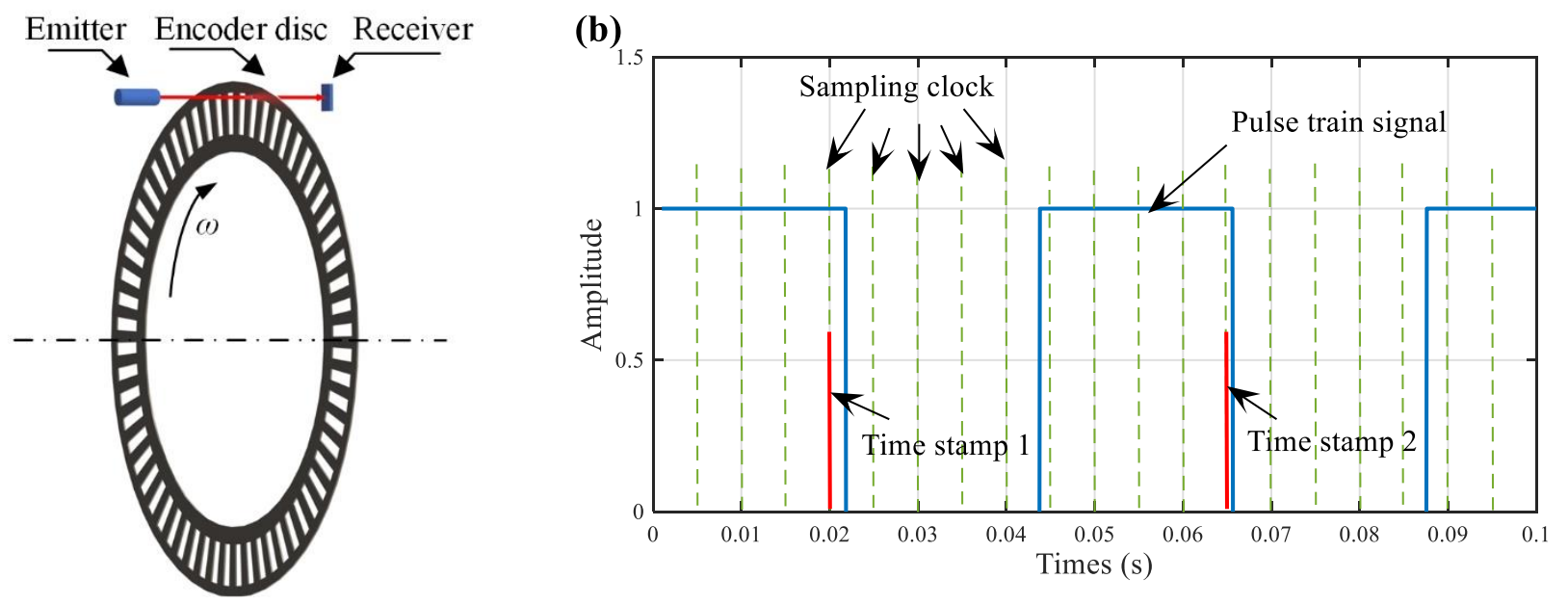

Fig. 1 (a) Typical setup to measure IAS and (b) illustration of generated pulse train from detector and time counting based method to estimate IAS

The IAS estimation methods can be classified into two categories: the time counting method and the FDD method. Time counting methods are based on principles like counting pulses in a given time, counting time between pulses [24] or polynomial fitting [25]. Time counting methods consider the raising and/or falling edge of the encoder pulse train signal as a time counting trigger. It assumes that the encoder is perfect with evenly divided slots. Thus, the IAS is estimated by dividing the division angle over the passage times, between the pulse edges. As illustrated in Fig. 1(b), the passage time highly depends on the sampling time stamp and the shaft rotating speed. It can only be an integer times of the sampling time interval. In these methods, the encoder errors and the corresponding false IAS are not considered. The IAS acquired by the counting methods is usually a pricewise signal, which is constant between time stamps. Therefore, a false infinite acceleration occurs at the time stamp. FDD method considers the encoder pulse train signal as a modulated signal, and the IAS is the derivative of the modulated phase $\theta(t)$ [11,12]. As discussed in [9][26], it has better signal to noise (SNR) compared to the time counting methods. This method extracts the instantaneous frequency from the analytic signal, which, in this case, is the encoder pulse train signal, and derivates it to obtain the IAS.

The analytical signal of the encoder output signal is given as[9][26]:

$$
a(t)=A(t) e^{i \theta(t)}
$$

Then, the IAS signal obtained by demodulation can be calculated as [9][26]:

$$
\dot{\theta}(t)=\operatorname{Im}\left[\frac{\dot{a}(t)}{a(t)}\right]
$$

where Im denotes the imaginary part of the complex signal.

\subsection{Encoder errors}

In theory, the slots on the encoder disc are evenly divided and the generated pulse train signal only contains mechanical signatures. However, due to inevitable manufacturing and adjustment tolerances, 
the distribution of the slots is non-uniform and slightly deviated from the ideal positions, leading to a false IAS at rotating harmonics. The rotary encoder accuracy can also be affected by encoder errors such as eccentric, tilt, distortion [14], graduation etc., and influence factors from the measurement system like quantification error [24], which are vital uncertain sources. Due to these errors, the measured angle $\theta^{\prime}$ is deviated a $\Delta \theta$ from the true rotating angle $\theta$. In [14], the influence of eccentric and tilt errors to a ring laser encoder, are studied based on the geometrical relationship in which the detector is perpendicularly placed to the rotating shaft. In this part, encoder errors with perpendicular as well as parallel detector are studied.

The encoder eccentric error denotes the distance of the encoder geometric centre deviated from the rotating centre, which generates a first-order component. According to the geometrical relationship[14][27], the measured encoder error $\Delta \theta_{e 1}$ and $\Delta \theta_{e 2}$ from a perpendicular[27] and parallel detector encoder can be given as:

$$
\begin{cases}\Delta \theta_{e 1} \approx \frac{\rho_{1} \sin \theta^{\prime}}{\rho_{1} \cos \theta^{\prime}-1}, & \theta^{\prime} \in[0,2 \pi) \\ \Delta \theta_{e 2} \approx \frac{\rho_{2} \sin \theta}{\rho_{2} \cos \theta-1}, & \theta \in[0,2 \pi)\end{cases}
$$

Therefore, their corresponding false IAS $\omega_{\mathrm{e} 1}(\mathrm{t})$ and $\omega_{\mathrm{e} 2}(\mathrm{t})$ is obtained as:

$$
\left\{\begin{array}{l}
\omega_{e 1}=\frac{\rho_{1}^{2}-\rho_{1} \cos \theta^{\prime}}{\left(\rho_{1} \cos \theta^{\prime}-1\right)^{2}} \omega(t) \\
\omega_{e 2}=\frac{\rho_{2}^{2}-\rho_{2} \cos \theta}{\left(\rho_{2} \cos \theta-1\right)^{2}} \omega(t)
\end{array}\right.
$$

where the subscript 1 and 2 represent perpendicular and parallel detector respectively, $\rho_{1}=\Delta r / r$ and $\rho_{2}=\Delta r /(r-\Delta r)$ are the eccentric ratio, $\omega(t)$ is the true IAS, $r$ is the encoder radius, and $\Delta r$ is the distance between the rotating centre and geometric centre.

The encoder tilt error denotes the angle between the encoder geometry central axis and the rotating axis, which generates a second-order component. The projection of the encoder geometry to the vertical shaft plane is an ellipse[27]. Accordingly, the tilt error and its corresponding false IAS with a tilt angle $\alpha$ are calculated as:

$$
\left\{\begin{array}{l}
\Delta \theta_{t}=\theta^{\prime}-\theta=\arccos \left(\frac{\cos \alpha \cos \theta}{\sqrt{(\cos \alpha \cos \theta)^{2}+(\sin \theta)^{2}}}\right)-\theta \\
\omega_{t}=\frac{d \Delta \theta_{t}}{d \theta} \frac{d \theta}{d t}=\left[\frac{\cos \alpha}{(\cos \alpha \cos \theta)^{2}+(\sin \theta)^{2}}-1\right] \omega(t)
\end{array}\right.
$$

Besides, the encoder graduation error can generate high-order components. According to the probability theory, the independent random deviations, graduation error, follow a normal distribution, namely the central limit theorem. The probability density of encoder graduation error $\Delta \theta_{\mathrm{g}}$ is given as: 


$$
f\left(\Delta \theta_{g} \mid \mu, \delta^{2}\right)=\frac{1}{\sqrt{2 \pi \delta}} e^{-\frac{\left(\Delta \theta_{g}-\mu\right)^{2}}{2 \delta^{2}}}
$$

where $\mu$ is the expectation of the distribution and $\delta$ is the standard deviation.

The combined periodical encoder error is expressed as:

$$
\Delta \theta_{p}(\theta)=\Delta \theta_{e}(\theta)+\Delta \theta_{t}(\theta)+\Delta \theta_{g}(\theta)=\sum_{n=0}^{N} a_{n} \sin \left(n \theta+\phi_{n}\right)
$$

where $N$ is the encoder resolution, $n$ is the $n^{\text {th }}$ order periodical component, $a_{n}$ is the amplitude of the $n^{\text {th }}$ order, $\phi_{n}$ is the initial phase of the $n^{\text {th }}$ order.

\subsection{Methods to suppress encoder errors}

To suppress the encoder errors and the corresponding false IAS, many methods have been proposed. These methods can generally be catalogued into two groups, namely cross-correction methods and self-correction ones. As discussed in Section 1, the self-correction methods are favoured as they don't need any reference device. Two self-correction methods, namely Resor method and Diamond method, are introduced here and set as reference methods to benchmark our proposed method in Section 2.4.

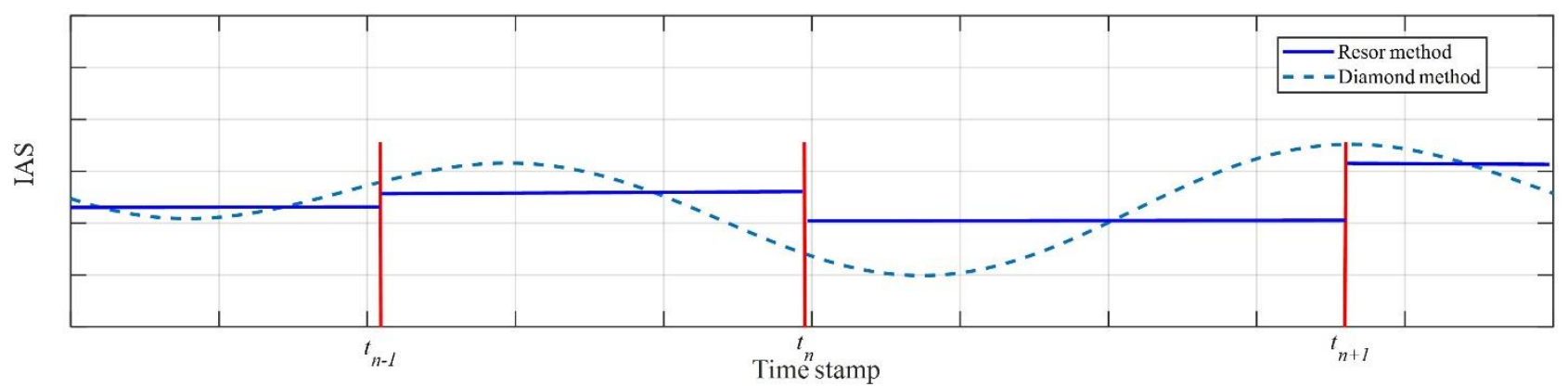

Fig. 2 IAS estimation methods with encoder error suppression

The basic idea of the Resor method [21] is that the unevenly distributed slots can be obtained and enhanced by the time synchronous averaging (TSA) process under constant speed. The time between time stamps, or passage time, corresponding to a certain slot should regress to the real passage time after TSA process. The processed passage time over the one revolution time reflects the real slot interval angle. A time domain resampling is then applied to this piecewise IAS to further eliminate the false IAS generated by the uneven time interval. For the $i^{\text {th }}$ revolution, the ratio of slot $n$ is simply calculated as[21]:

$$
r_{\text {Resor }}(n, i)=\frac{t_{(i-1) N+n+1}-t_{(i-1) N+n}}{T_{i}}
$$

where $T_{i}$ is the pastime of $i$ revolution, $N$ is the slot number of the encoder 
It assumes the shaft rotating at a constant speed, therefore, it also mistakes the true IAS fluctuation of shaft as a part of encoder error.

The Diamond method is based on the concept that the IAS between adjacent time stamps is continuous and can be expressed by a second-order polynomial function. The author also indicates that the IAS at the time stamps should also be continuous despite belonging to different time subdomain, as shown in Fig. 2. Based on these assumptions, a matrix with angle interval and polynomial coefficient [23] can be constructed. For the $i^{\text {th }}$ revolution, the continuous second-order polynomial IAS between slot $n$ and $n+1$ is given as[23]:

$$
\dot{\theta}_{n, i}(t)=a_{n, i}\left(t-t_{(i-1) N+n}\right)^{2}+b_{n, i}\left(t-t_{(i-1) N+n}\right)+c_{n, i} \quad t_{(i-1) N+n} \leq t \leq t_{(i-1) N+n+1}
$$

It is assumed that the parameters in the matrix follow multivariate Gaussian distribution, therefore, a Bayesian linear regression is applied to acquire more accurate parameters. However, it is a timeconsuming process to acquire a convergent regression result, especially with long data length.

\subsection{IAS estimation based on a dual detector setup}

Instead of using one detector, a setup to include dual detectors is employed in this paper, as shown in Fig. 3. Two detectors, illustrated as $\# 1$ and $\# 2$, are placed in parallel with an arbitrary angle $\beta$. Suppose the acquired angle measurement includes a true shaft rotating angle $\theta$ and false deviations $\theta_{d}$ caused by encoder error, the measured encoder error deviations $\theta_{d 1}(\theta)$ from \#1 detector and $\theta_{d 2}(\theta)$ from \#2 detector are the same with a $\beta$ phase shift, i.e. $\theta_{d}(\theta)=\theta_{d 1}(\theta)=\theta_{d 2}(\theta-\beta)$. The encoder errors can be directly eliminated as in Section 2.4.2.

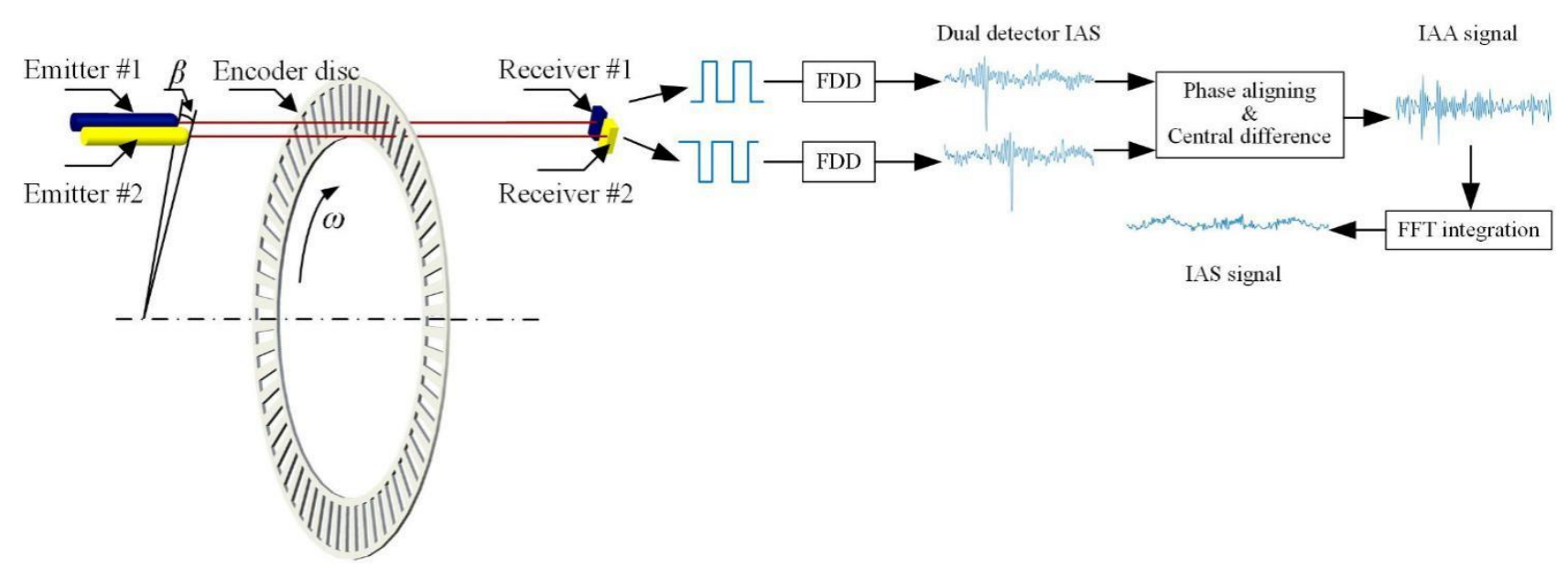

Fig. 3 Illustration of IAS measurement with dual optical detector

Under conditions with constant or small fluctuating speed, the measured shaft rotating angle $\theta^{\prime}(t)$ can be assumed as $\theta^{\prime}(t) \approx \omega t$. Therefore, the measured angle from \#1 and \#2 detector in the time domain can be expressed as: 


$$
\left\{\begin{array}{l}
\theta_{1}^{\prime}(t)=\theta(t)+\theta_{d}(\theta(t)-\beta) \\
\theta_{2}^{\prime}(t)=\theta(t)+\beta+\theta_{d}(\theta(t))
\end{array}\right.
$$

Therefore, the phase difference between the two measurements are purely caused by the encoder errors:

$$
\theta_{1}^{\prime}(t)-\theta_{2}^{\prime}(t)=\theta_{d}(\theta(t)-\beta)-\theta_{d}(\theta(t))-\beta
$$

To eliminate the encoder errors and acquire a phase difference with the true shaft rotating, the dual detector signals are aligned by shifting signal from $\# 2$ detector with $\Delta t=\beta / \omega$, and is given as:

$$
\theta_{1}^{\prime}(t)-\theta_{2}^{\prime}(t-\Delta t)=\theta(t)-\theta(t-\Delta t)-\beta
$$

Based on the above analysis, an algorithm to estimate IAS signal is proposed in Fig. 4, which includes two key steps, with the first step to estimate encoder error and the second step to estimate the true IAS signal. Detailed implementation of these two steps will be explained in the following two subsections.

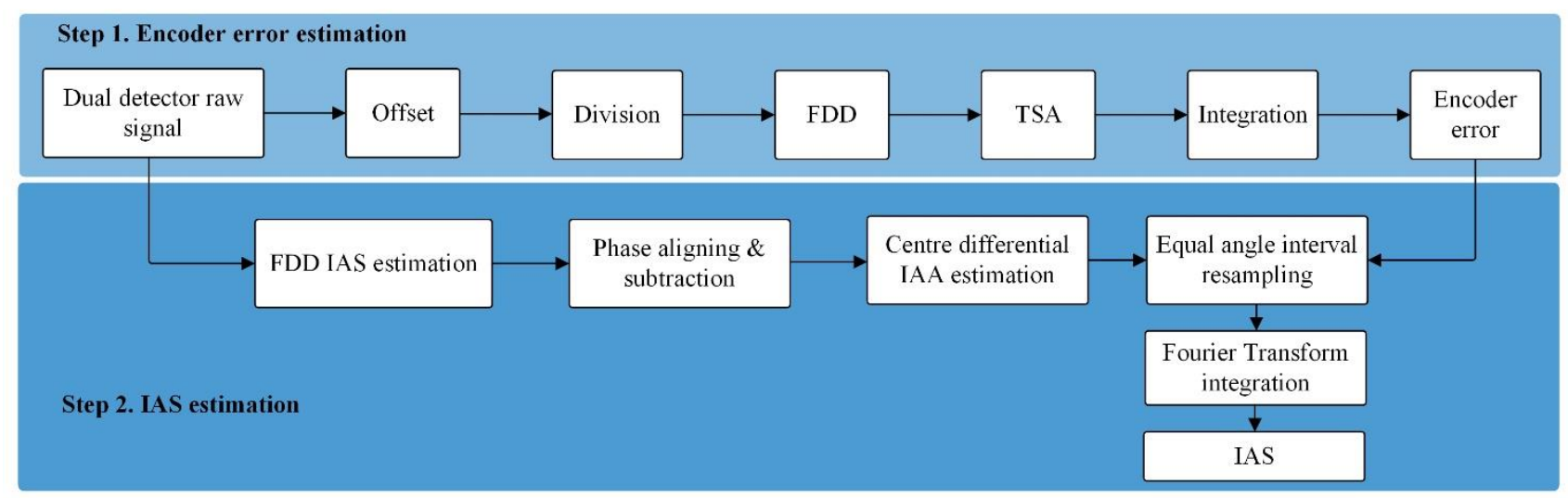

Fig. 4 Dual detector IAS estimation flow chart

\subsubsection{Encoder error estimation}

After acquiring the raw signals from the two detectors, the first step is to estimate the encoder errors. The detail of this process is described by the following steps:

(I.1) Offset the raw pulse train signals and ensure the signals are positive only, which avoids phase distortion when the dual channel signals perform division operation in the next step.

(I.2) Divide the processed two channel signals in Step (I.1) to obtain a phase difference signal which only contains encoder deviations information.

(I.3) Demodulate the phase difference signal to acquire an false IAS signal, as in Eq.(14). 
(I.4) Apply TSA to the false IAS with the shaft rotating period as the averaging period. Here the TSA process acts like a comb filter [28], which enhances the chosen period components, whilst suppressing the random and non-integer period components.

(I.5) Integrate the demodulated encoder deviation phase difference IAS to estimate the encoder error deviations.

The analytical signal of the phase difference signal in Step (I.2) is given as:

$$
a_{d}(t)=\frac{a_{1}(t)}{a_{2}(t)}=\frac{A_{1}(t)}{A_{2}(t)} e^{i\left[\theta_{1}^{\prime}(t)-\theta_{2}^{\prime}(t)\right]}
$$

According to Eq. (2) and (13) and the central difference, i.e. the demodulated two detector IAS difference, in Step (I.3) is given as:

$$
\dot{\theta}_{d}\left(t-\frac{\Delta t}{2}\right) \approx \frac{d\left[\theta_{d}(t-\Delta t)-\theta_{d}(t)\right]}{d t}=\frac{d\left[\theta_{1}^{\prime}(t)-\theta_{2}^{\prime}(t)\right]}{d t}=\operatorname{Im}\left[\frac{\dot{a}_{d}(t)}{a_{d}(t)}\right]
$$

Under conditions with constant or small fluctuating speed, the interval angle $\Delta \theta$ can be approximately estimated as $\Delta \dot{\theta} \Delta t$. Theoretically, as in Eq. (11) and (14), the true IAS signals from the dual channel are cancelled, while the central difference of the encoder deviation is kept. Therefore, according to the Eq.(14), the encoder deviation angle, i.e. the encoder error signal, can be approximately calculated as:

$$
\theta_{d}(t) \approx \dot{\theta}_{d}(t) \Delta t \approx-\operatorname{Im}\left[\frac{\dot{a}_{d}\left(t+\frac{\Delta t}{2}\right)}{a_{d}\left(t+\frac{\Delta t}{2}\right)}\right] \Delta t
$$

In the result of the central differential operation, the encoder deviation angle is estimated at the centre, namely $t+\Delta t / 2$.

\subsubsection{IAS estimation}

After estimating the encoder errors, the true IAS can be estimated by the following key steps:

(II.1) Shift the demodulated IAS from \#2 detector with a phase $\beta$ so that it aligns with that from \#1 detector.

(II.2) Subtract between the aligned two IAS signals to cancel out the false IAS. Under constant or quasi-constant condition, the false IAS from dual channels are considered to have a phase shift, thus the error can be removed by such a simple subtraction. In the meantime, the residual signal is a difference of the dual channel true IAS signals.

(II.3) Apply the central difference method to the difference of the dual channel true IAS signals, which yields an estimate of the instantaneous angular acceleration (IAA) signal. 
(II.4) Resample the IAA signal with equal angle interval, which excludes the false components at multiple shaft rotating orders due to uneven angular sampling [21].

(II.5) Integrate the IAA signal through a frequency domain method to obtain an IAS signal.

According to Eq. (2), (10) and (12), the measured IAS contains both the true shaft rotating IAS and the false IAS. Therefore, the phase difference only containing true IAS information, as in Step (II.2), is given as:

$$
\dot{\theta}_{1}^{\prime}[\theta(t)]-\dot{\theta}_{2}^{\prime}[\theta(t)-\beta]=\dot{\theta}[\theta(t)]-\dot{\theta}[\theta(t)-\beta]
$$

Moreover, the IAA from central difference method, as in Step (II.3), is approximately calculated as:

$$
\ddot{\theta}\left[\theta(t)-\frac{\beta}{2}\right] \approx \frac{\dot{\theta}[\theta(t)]-\dot{\theta}[\theta(t)-\beta]}{\Delta t}
$$

Based on the Fourier transform characteristic, the true IAS is expressed as:

$$
\dot{\theta}\left(t-\frac{\Delta t}{2}\right)=F^{-1}\left(\frac{F\left(\ddot{\theta}\left(t-\frac{\Delta t}{2}\right)\right)}{j \omega e^{j \omega \frac{\Delta t}{2}}}\right)
$$

where $F$ denotes the Fourier transform and $F^{-1}$ denotes the inverse Fourier transform.

The central difference method has a second-order accuracy, which depends on the step size $\Delta t / 2$ as in Eq.(18). The proposed method eliminates false IAS directly and applies the central difference to estimate the true IAS.

\section{Numerical study}

In Section 2, a method to estimate IAS signal based on a dual detector setup is proposed. In this Section, a numerical study is performed to evaluate the effectiveness of the proposed method to minimise/eliminate encoder errors and also the accuracy of the estimated IAS influenced by the angle between the dual detector and the shaft rotating speed.

\subsection{Simulation of measured signals with a dual detector setup}

To evaluate the proposed method, a typical numerical encoder signal $s_{1}(t)$ acquired by detector \#1 are simulated with consideration of the typical encoder errors, as defined by: 


$$
s_{1}(t)=\frac{4}{\pi} \sum_{k=1}^{H} \frac{1}{2 k-1} \sin \left[\begin{array}{l}
N \omega t+\int \sum_{n=1}^{N_{1}} a_{n} \cos \left(2 \pi n f_{r} \tau\right) d \tau+\int \sum_{n=1}^{N_{2}} b_{n} \cos \left(2 \pi n f_{c} \tau\right) d \tau \\
+\int \sum_{n=1}^{N_{3}} c_{n} \cos \left(2 \pi n f_{r} \tau\right) d \tau
\end{array}\right]
$$

where $N$ is the encoder resolution, $\omega$ is the shaft rotating frequency in $\mathrm{rad} / \mathrm{s}, a_{n}, b_{n}$ and $c_{n}$ are the corresponding Fourier series amplitudes; $f_{r}$ represents the shaft rotating frequency in $\mathrm{Hz}, f_{c}$ represents a characteristic frequency of the rotating system.

In the simulated encoder signal in Eq.(19), the pulse train carrier wave is simulated by a summation of a series of odd-order sinusoidal waves. The shaft rotating frequency, typical characteristic frequency and encoder errors are reflected by changes in the phase of the pulse train carrier wave. In specific, the phase modulated terms in Eq.(19) correspond respectively to the shaft rotating speed, true fluctuations from the shaft rotating speed and its harmonics, true fluctuations from the characteristic frequency and its harmonics, and the false fluctuations caused by encoder errors. The encoder errors are simulated as a summation of Fourier series with the fundamental frequency being the same as the shaft rotating frequency, based on the analysis in Section 2.2.

According to the discussion in Section 2, the true fluctuations from the two detectors are the same while the false fluctuations from them have a phase shift. Therefore, the encoder signal $s_{2}(t)$ acquired by detector $\# 2$ is simulated as:

$$
s_{2}(t)=\frac{4}{\pi} \sum_{k=1}^{H} \frac{1}{2 k-1} \sin \left[\begin{array}{l}
N \omega t+\int \sum_{n=1}^{N_{1}} a_{n} \cos \left(2 \pi n f_{r} \tau\right) d \tau+\int \sum_{n=1}^{N_{2}} b_{n} \cos \left(2 \pi n f_{c} \tau\right) d \tau \\
+\int \sum_{n=1}^{N_{3}} c_{n} \cos \left(2 \pi n f_{r} \tau-\Delta t\right) d \tau+\beta
\end{array}\right]
$$

where $\beta$ is the angle between the two detectors and $\Delta t$ is the time delay due to the angle $\beta$.

According to Eq.(4) and Eq.(5), the encoder errors and the corresponding false IAS amplitudes are approximately proportional to $\rho$ eccentric ratio, $1-\cos \alpha$ related to encoder tilt and $1 / \sqrt{2 \pi \delta}$ related to encoder manufactory tolerance. For eccentric error $\rho=0.01$, a false IAS with $0.01 \omega$ amplitude is simulated. For $\alpha=1^{\circ}$, a false IAS with $0.00015 \omega$ amplitude is simulated. Therefore, the variable values in Eq.(19) and (20) are set as in Table 1, which are set to be in consistent with the measurements from the experimental system in Section 4.

Table 1. Variable values of the simulated encoder signal

\begin{tabular}{lllllllr}
\hline \multicolumn{2}{l}{ Constant term } & Integer & \multicolumn{3}{c}{ Non-integer } & \multicolumn{3}{c}{ False } \\
\hline$N$ & 60 & $a_{1}$ & $0.01 \mathrm{rad} / \mathrm{s}$ & $b_{1}$ & $0.018 \mathrm{rad} / \mathrm{s}$ & $c_{1}$ & $0.03 \mathrm{rad} / \mathrm{s}$ \\
$\omega$ & $114.8 \mathrm{rad} / \mathrm{s}$ & $f_{r}$ & $18.27 \mathrm{~Hz}$ & $b_{2}$ & $0.048 \mathrm{rad} / \mathrm{s}$ & $f_{r}$ & $18.27 \mathrm{~Hz}$ \\
$\beta$ & $3^{\circ}$ & & & $f_{c}$ & $47.2 \mathrm{~Hz}$ & & \\
\hline
\end{tabular}



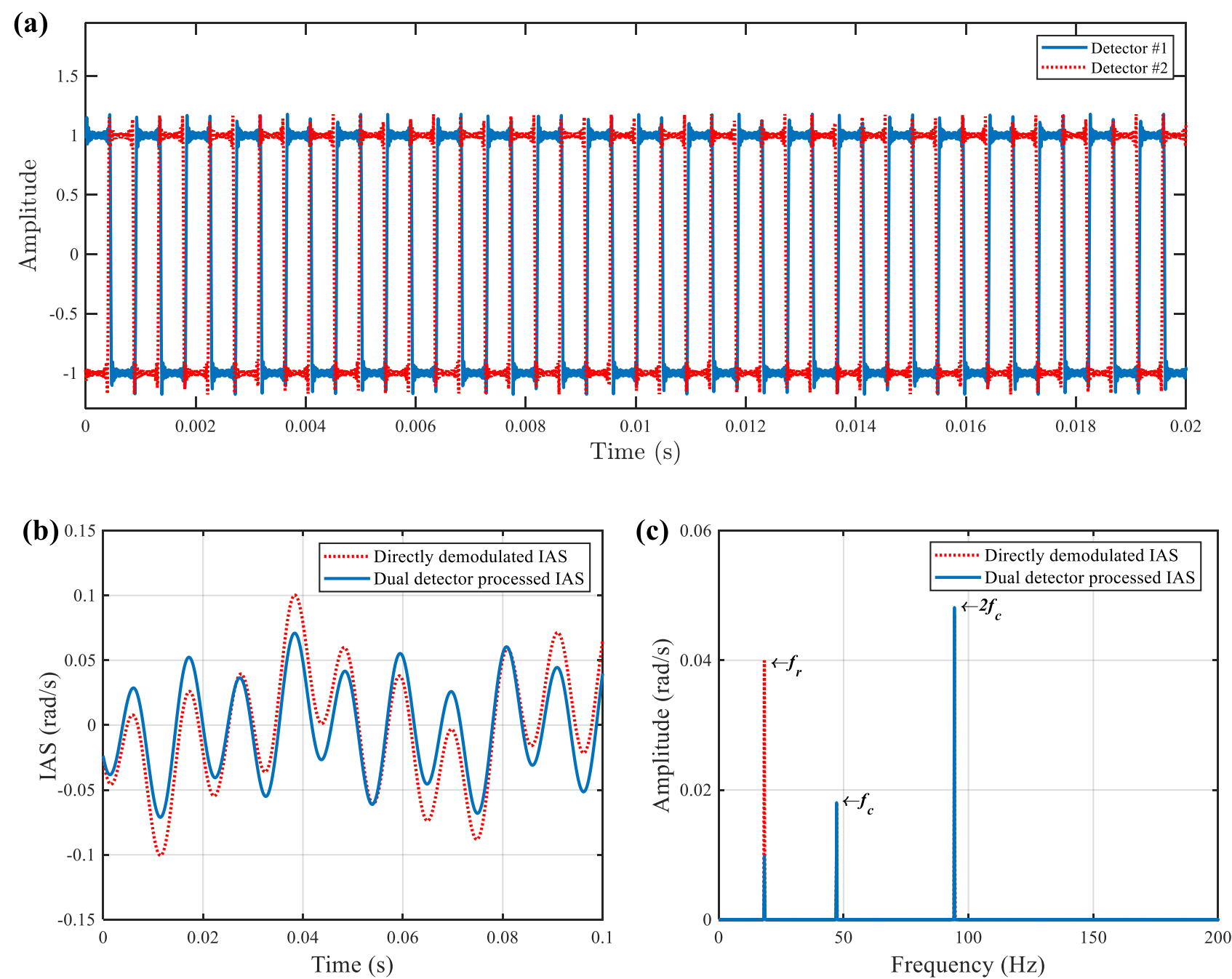

Fig. 5 Evaluation of the dual detector method: (a) IAS waveform and (b) its spectrums

The simulation results are presented in Fig. 5, in which Fig. 5 (a) shows the acquired encoder signals from detector \#1 and \#2. Only the torsional oscillation part of the IAS is shown as in Fig. 5, because the rigid-body-motion part of the IAS only reflect the constant rotating speed term. Due to the angle $\beta$ between detectors, a significant time delay is observed in encoder signal of \#2. It can be seen that the proposed method successfully extracts the true IAS from the measured IAS, or directly demodulated IAS signals, especially at the rotating frequency $f_{r}$ where a true IAS has an amplitude of $0.01 \mathrm{rad} / \mathrm{s}$, being the same as the simulated one. The direct demodulated IAS show significantly higher amplitude than expected as shown in Fig. 5 (c).

\subsection{IAS estimation accuracy influence factors}

The estimation of the IAS signal can be affected by a variety of factors, like the estimation methods, measurement system parameters and also the dual detector setup parameters in our case. As discussed in Section 1, the FDD method performs better than the counting method and the local polynomial fitting method. The influence by measurement system parameters and the dual detector setup parameters will be discussed below. 


\subsubsection{Measurement system parameters}

Parameters like rotating speed, sampling rate, time duration and encoder resolution decide the performance of the measurement system. These parameters interact with each other and are restricted by the register bit, maximum sampling frequency $F_{S}$, and channel numbers of the hardware. As the total data length is limited, choosing a higher sampling rate means that the recording time duration has to be truncated. Higher sampling frequency suppresses the encoder quantification errors [24] and the background noise in spectrum [22], meanwhile decreasing the frequency resolution. According to the Nyquist sampling theorem, the encoder resolution to interested event frequency per revolution ratio should be larger than 2. In [24], a ratio of 20 is recommended for the counting based method. In [9], the sampling frequency recommended 4 times higher than the IAS maximum interested frequency, and concluded that higher encoder resolution and longer time duration lead to a lower noise level and improved IAS estimation accuracy.

The IAS estimation error of the counting method, due to quantification error, is proportional to the product of the shaft IAS and the encoder resolution, which is predicted as [24]:

$$
\omega_{q}(t)= \pm \frac{\omega(t) N}{60 F_{s}}
$$

\subsubsection{Dual detector parameters}

The dual detector parameter influences are illustrated in Fig. 6. The central difference step size is the main influence factor with two aspects: interval angle and rotating speed. In angle domain, the central difference step size is $\beta / 2$, whilst the step size in time domain is $\beta / 2 \omega$. To study the parameter influence in the dual detector process, an true IAS signal, which in frequency domain is a sum of the $0.2 \mathrm{n} f$, $\mathrm{n}=1,2, \ldots, 300$ harmonics with same $0.03 \mathrm{rad} / \mathrm{s}$ amplitude, is considered and processed by dual detector method with different parameters. The order spectrum amplitude is normalized by dividing $0.03 \mathrm{rad} / \mathrm{s}$. In Fig. 6 (a), the shaft speed is $115 \mathrm{rad} / \mathrm{s}$, while the interval angle varied. It can be seen that the interval angle is similar to a low-pass filter. In other words, the smaller the interval angle, the higher the lowpass filter stop band. The proposed method directly eliminates the encoder error, but it also suppresses the higher frequency component of true IAS due to the low-pass filter effect, where the Fig. 6 (a) indicate the true IAS.

The interval angle $\beta$ to encoder division angle, $2 \pi / N$, ratios are $0.03,0.17,0.5,1,1.7,15$ separately as in Fig. 6 (a). It is noticed that the IAS order spectrum with angle ratio 0.17 is nearly flat. In Fig. 6 (b), the angle ratio is 0.5 , while the shaft speed varied. The higher the speed, the flatter the IAS spectrum. The estimation accuracy highly depends on the angle interval, while it is less influenced by the speed. 

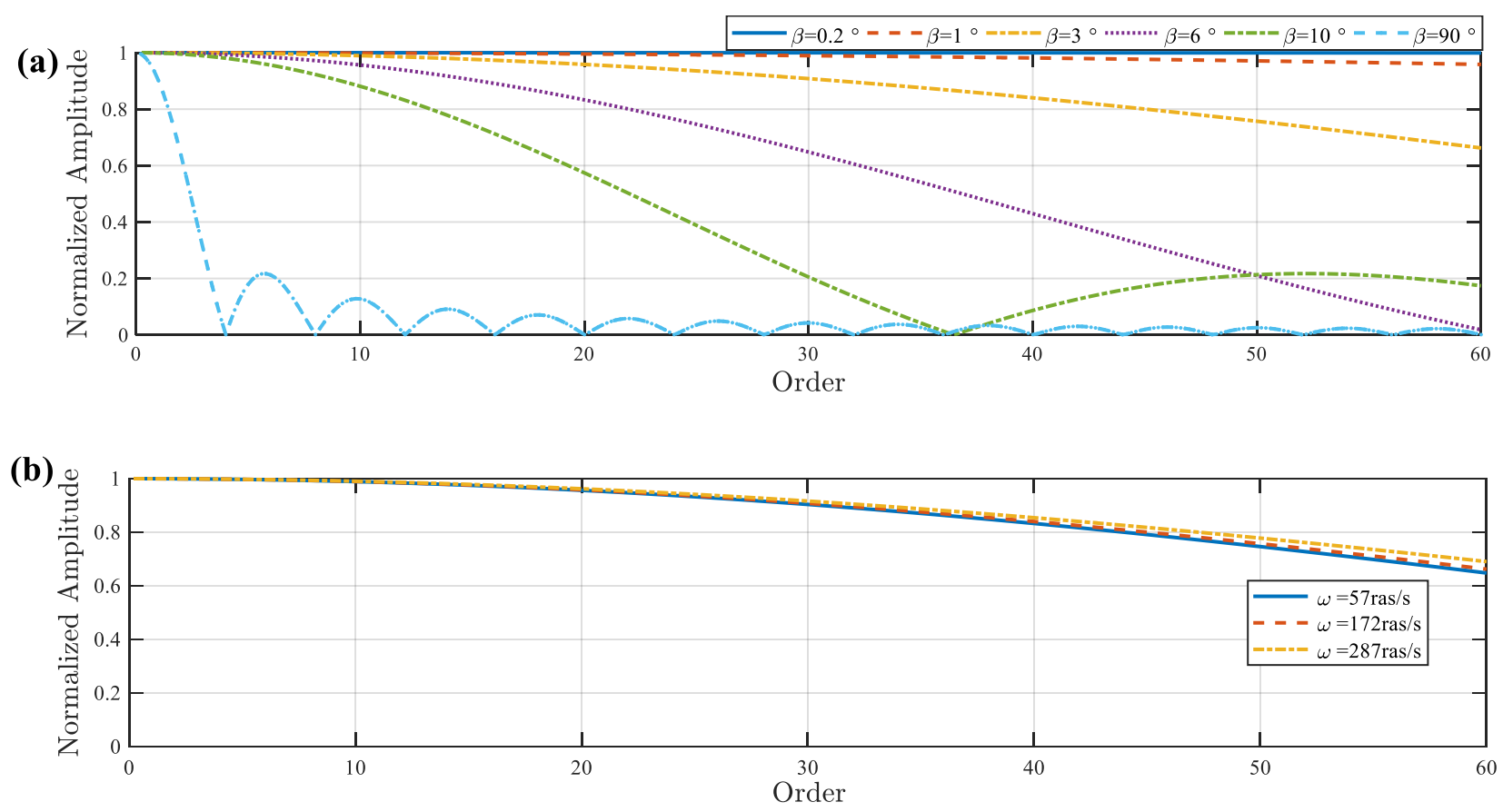

Fig. 6 Order spectrum of estimated IAS influenced by (a) the angle between two detectors and (b) the shaft rotating speed

\section{Experimental validation}

To evaluate the performance of the proposed IAS estimation method in practice, an encoder disc is 3D printed and mounted a planetary gearbox test rig. The acquired encoder signals are processed by our proposed method in Section 2.4, as well as the Resor method and the Diamond method for comparison. Besides, only the IAS fluctuations are studied in this section. The constant IAS part is not particularly analysed, due to that it has a much larger value and does not reflect the mechanical dynamics.

\subsection{Experimental setup}

The test rig mainly consists of an AC motor (11 kW, full speed $1500 \mathrm{r} / \mathrm{min}$ ) that drives the whole test rig, a DC generator ( $85 \mathrm{~kW}$ is full speed $1850 \mathrm{r} / \mathrm{min}$ ), that applies torque to the system and transforms the energy into electrical energy, two back-to-back mounted planetary gearboxes (PG). A 1.23 overall ratio is required to make the most efficient use of these two components. Hence, the transmission ratio of PG 1 and PG 2 are 5.77 and 7.2 separately. The PG 1 jointed with AC Motor acted as a reduction gearbox, while the PG 2 jointed with the DC generator increased the speed. Two PGs are utilized to adjust the speed of AC motor and DC generator. In this case, the generated DC power was consumed by the resistance bank. These mechanical components are connected together by three rubber tyre couplings, which suppress the potential torsional vibration transferring through the shafts. The maximum load at the low-speed shaft, between the PGs, is $400 \mathrm{Nm}$, limited by the rubber coupling. 
Moreover, the test rig is controlled by an open loop system, in which the speed is controlled by setting parameters rather than timely speed feedback. Therefore, the real speed of the test rig slightly deviated from the aim speed.

The encoder is attached to the high-speed shaft of PG 2 and close to the DC generator, as illustrated in Fig. 7 (a). The encoder includes two parts: an encoder disc and two stationery detectors. The detector are placed with an interval angle $\beta=3.26^{\circ}$ and parallel to the shaft axis. Note that $\beta$ is calculated by the interval angle pass time over the one revolution pass time, and then averaged. The encoder disc, Fig. 7 (b), is 3D printed in two parts. The two parts are bolted together and attached to the shaft. The encoder disc has 60 equally divided slots in total, allowing the laser beam to pass or be blocked.

(a)

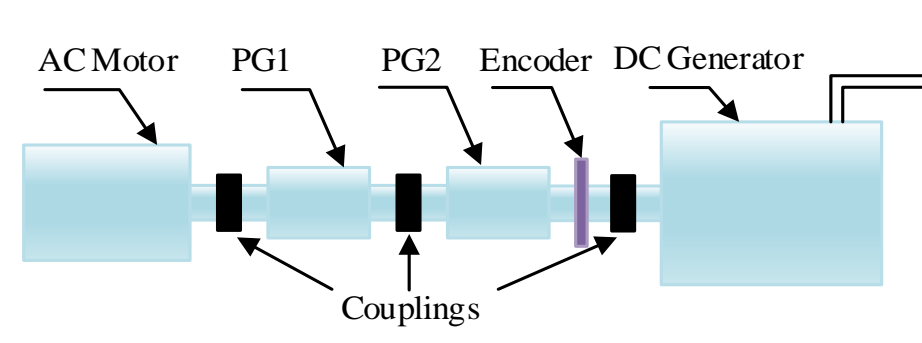

Resistance bank

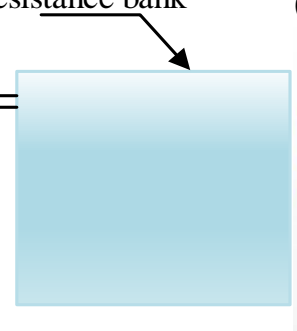

(b)

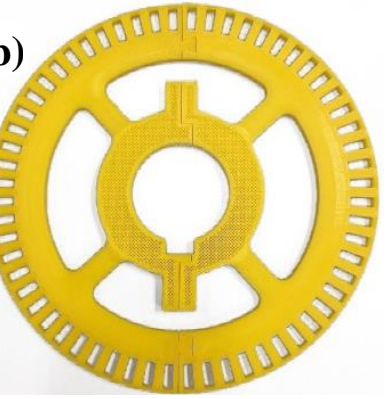

Fig. 7 The test rig (a) schematic (b) 3D printed encoder disc

In this paper, the $\mathrm{AC}$ motor was set running at $60 \%$ of the full speed, namely $900 \mathrm{r} / \mathrm{min}$, and the DC generator applied $90 \%$ of the full load, namely $360 \mathrm{Nm}$. The dual channel signals were simultaneously recorded with sampling rate at $96 \mathrm{kHz}$ and time duration of 40 seconds. The characteristic frequency of the PG 2 included mesh frequency, rotating frequencies and their harmonics. PG 2 included three planet gears, a sun gear, a ring gear and a planet carrier. The tooth number of the PG 2 were: the ring gear $Z_{r}=62$, the planet gear $Z_{p}=26$ and the sun gear $Z_{s}=26$. Under this operating condition, the rotating frequency of the sun gear was $18.8 \mathrm{~Hz}$ rather than the theoretical $18.7 \mathrm{~Hz}$, because of the applied $90 \%$ load. The signature frequencies of rotating events such as the carrier rotating frequency $f_{c}$, the planet gear rotating frequency $f_{p}$, the sun gear rotating frequency $f_{s}$ and the mesh frequency $f_{m}$ are listed in Table 2. Moreover, the other relative rotating frequencies to the carrier shaft such as the sun gear relative rotating frequency $f_{s r}$, planet gear relative rotating frequency $f_{p r}$ are also provided. When rotating, the sun gear passes by three planet gears in sequence in one sun gear revolution, the sun gear pass frequency, or sun gear fault frequency, $f_{s p}=3 f_{s r}$. Each planet passes by the sun gear and the ring gear in one planet revolution, the corresponding pass frequency, or the planet fault frequency, $f_{p p}=2$ $f_{p r}$. Those frequencies are listed in Table 2.

Table 2. PG 2 characteristic frequencies

\begin{tabular}{cccccccc}
\hline$f_{c}$ & $f_{s}$ & $f_{s r}$ & $f_{s p}$ & $f_{p}$ & $f_{p r}$ & $f_{p p}$ & $f_{m}$ \\
\hline $2.61 \mathrm{~Hz}$ & $18.81 \mathrm{~Hz}$ & $16.2 \mathrm{~Hz}$ & $48.6 \mathrm{~Hz}$ & $3.6 \mathrm{~Hz}$ & $6.2 \mathrm{~Hz}$ & $12.5 \mathrm{~Hz}$ & $161.3 \mathrm{~Hz}$ \\
\hline
\end{tabular}




\subsection{Encoder error estimation}

The encoder slots are usually deviated from their ideal positions due to various encoder errors. These deviations lead to significant jitters in both the time and frequency domains [21]. Resor method is chosen as a reference based on its small standard deviation [23]. The reference encoder error estimation archived using the conventional time counting method. It obtains the circumference ratio of each encoder slots by calculating the ratio of the averaged encoder slot passing time to averaged one revaluation pass time.
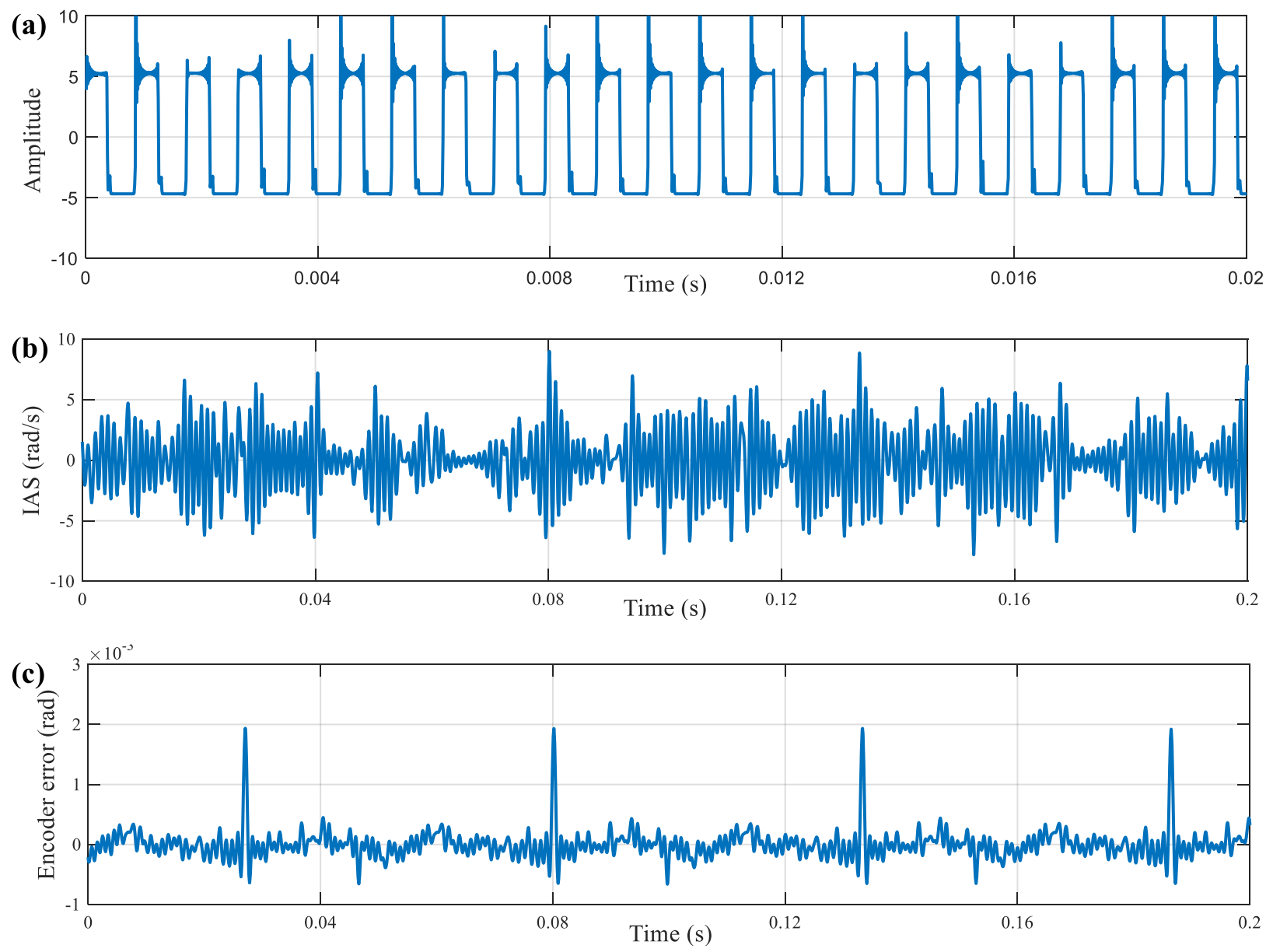

Fig. 8 Encoder error estimation (a) divided signal, (b) phase demodulated signal, and (c) error signal

Fig. 8 (a) illustrates a segment of the phase difference signal obtained by the division between the two signals from dual channels, detailed by Step (I.2) in Section 2.4.1. It can be seen that several peaks appears at the rising and falling edges of the square waves due to the jitter effect of low pass filter. The phase demodulated signal in Step (I.3) is illustrated as shown in Fig. 8 (b). It shows strong randomness and could not show up the basic periodicity of encoder error. By applying TSA to the phase modulated signals according to Step (I.4) and implementing the integration of Step (I.5), a signal is obtained as shown in Fig. 8 (c). The periodic behaviour can now be clearly presented and hence can be taken as the signal in association with encoder errors. 

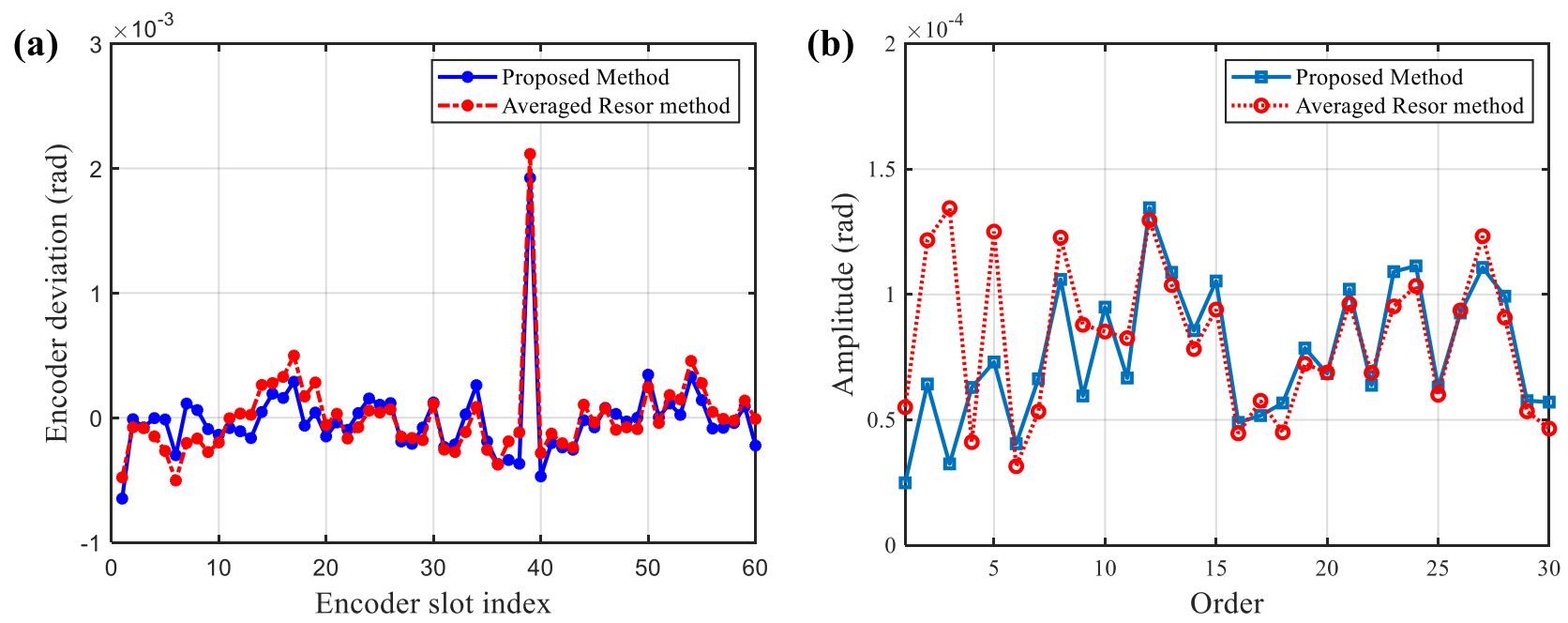

Fig. 9 Encoder deviations from Resor and proposed methods (a) deviation (b) spectrum
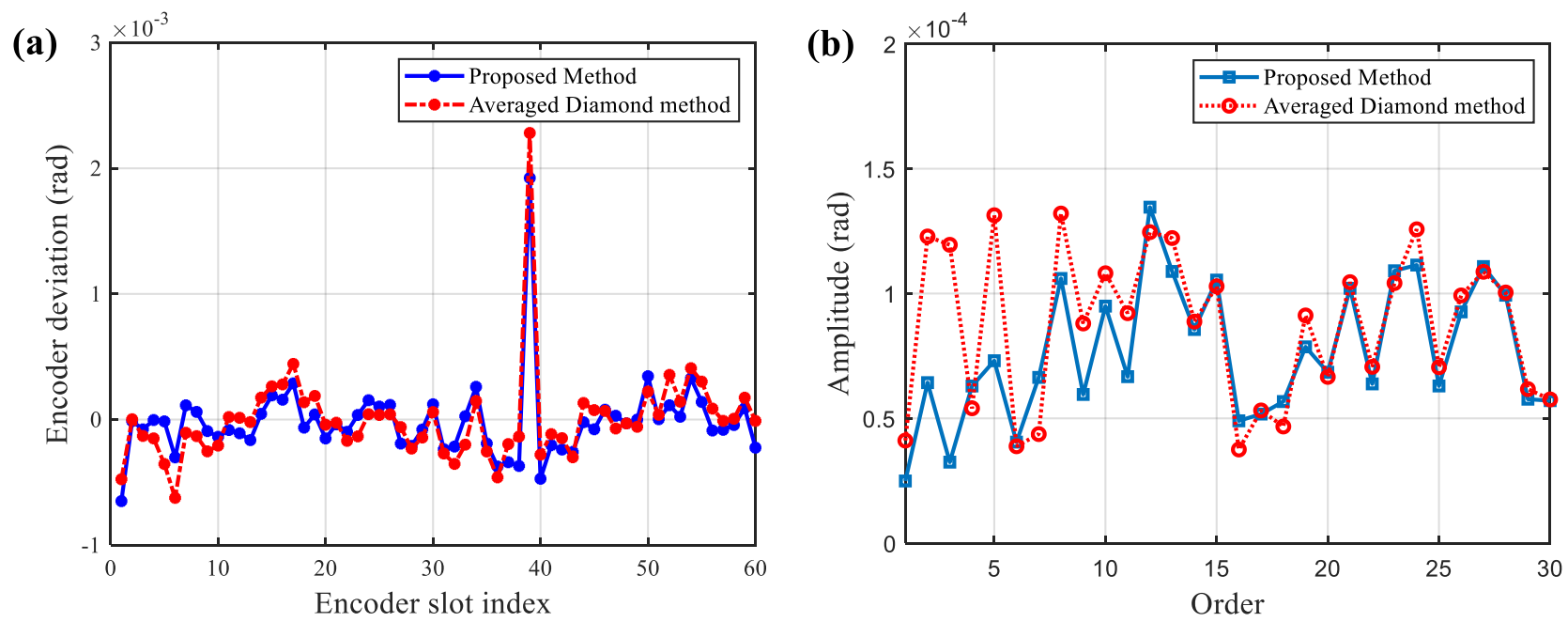

Fig. 10 Encoder deviations from Diamond and proposed methods (a) deviation (b) spectrum

For a comparative study, Fig. 9 and Fig. 10 presents one period of the encoder deviation, or encoder error signals, from proposed method, Resor method and Diamond method. The encoder error signal in Fig. 8 (c) is resampled on to the encoder index to corresponding to the encoder deviation results from Resor and Diamond. Proposed method considers the encoder error from both detector and obtains a comprehensive encoder error. The encoder error from \#1 and \#2 estimated by Resor method amd Diamond method are slightly different. Hence, two detector encoder errors are averaged to represent the comprehensive encoder error. Encoder deviations of proposed method have a good coherence with two encoder deviations of reference methods, which indicates the efficient estimation of the proposed method.

As explained in Section 2.2, the encoder errors can be expressed in a Fourier series. Accordingly, the $n^{\text {th }}$ order amplitude indicates the corresponding order encoder errors, in which the first order represents the eccentric error and the second order represents the tilt axis error. The proposed method's encoder deviations spectrums, as in Fig. 9 (b) and Fig. 10 (b), has a good coherence with reference methods' 
IAS spectrum, especially for the ones higher than $6^{\text {th }}$ order. The high energy at lower orders of reference methods may because that the reference methods consider the true low order shaft IAS fluctuations as part of encoder error.

\subsection{IAS estimation}

After getting the encoder error, the false IAS in the measurement is supressed, hence, the true IAS can be obtained. The conventional IAS estimation methods, namely time counting and FDD methods, measure an IAS signal with encoder errors. In Fig. 11 (a), the measured IAS waveforms are illustrated. The marked jitters 1,2,3,4 are periodical, which are generated by the encoder error occurs at slot $39^{\text {th }}$ as in Fig. 9 and Fig. 10. It is noticed that the FDD method estimates an IAS with high order oscillations, which may be caused by overfitting. In Fig. 11 (b), the IAS frequency spectra are illustrated. It can be seen that the false IAS at shaft rotating harmonics are dominant, the FFD method has a lower noise, which reveals more frequency components than the time counting method.

(a)
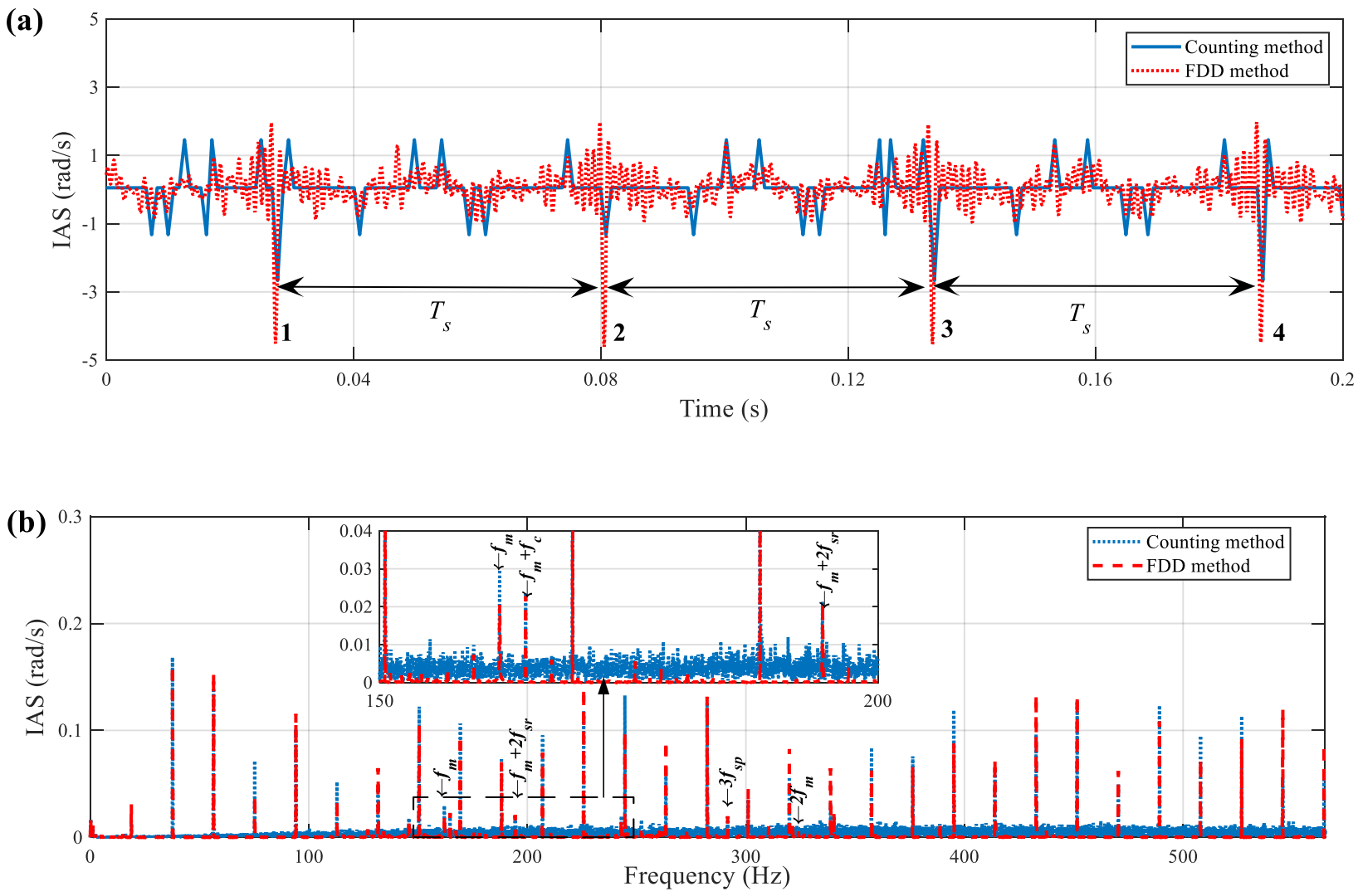

Fig. 11 Conventional methods estimated IAS (a) waveform (b) spectra

The comparison plots of the IAS signals obtained from the three error suppression methods are shown in Fig. 12 and Fig. 13. The IAS regressed by Diamond method is not perfect, due to the IAS discontinuity at time stamps. This is caused by the quantification errors due to the insufficient sampling rate and relatively high rotating speed. The Diamond IAS illustrated bellow is calculated by its regressed angle interval over corresponding pass time. 
The Fig. 12 illustrates the IAS oscillation waveforms of the three signals. Ideally, the test rig runs at a constant speed. However, due to excitation factors such as load variation, mesh stiffness variation, shaft unbalance and gear eccentric, the true shaft IAS fluctuates with a small amplitude. In [23], authors concluded that constant IAS is approximately linear with small deviations in practice. To quantitatively analyse the IAS deviations, the IAS standard deviation and kurtosis of the three methods are listed in Table 3Error! Reference source not found.. It can be seen that the proposed method has the smallest standard deviations value, 0.24 , and the smallest kurtosis value, 2.72 . The waveforms, as illustrated in Fig. 12, of the dual detector processed IAS is smoother with fewer glitches, indicating the proposed method is a more accurate one. Despite improvements in Resor and Diamond methods, the estimated IAS by these two methods still show obvious jitters, as shown in Fig. 12, which are mainly caused by time quantification error.

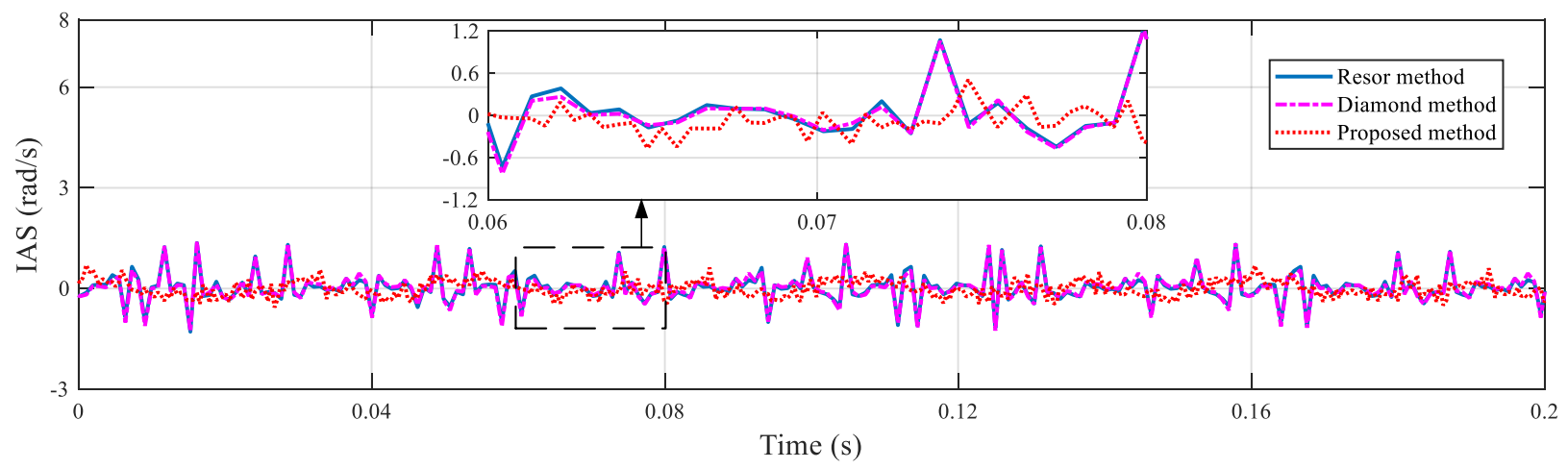

Fig. 12 IAS waveforms of the Resor method, Diamond method and the proposed method

Table 3. IAS deviation evaluation parameters

\begin{tabular}{lccccc}
\hline & Counting method & FDD method & Resor method & Diamond method & Dual detector method \\
\hline Standard deviation & 0.63 & 0.59 & 0.47 & 0.48 & 0.24 \\
Kurtosis & 8.46 & 18.13 & 4.99 & 5.06 & 2.72 \\
\hline
\end{tabular}

(a)

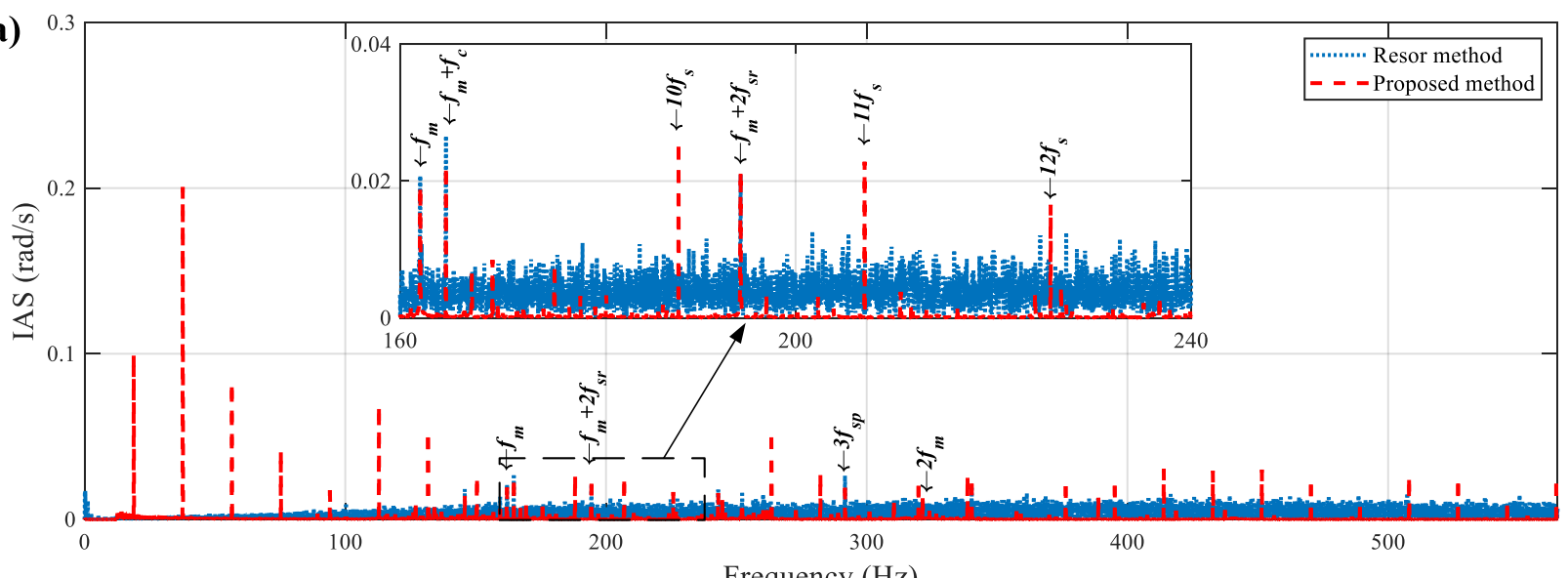

Frequency $(\mathrm{Hz})$ 


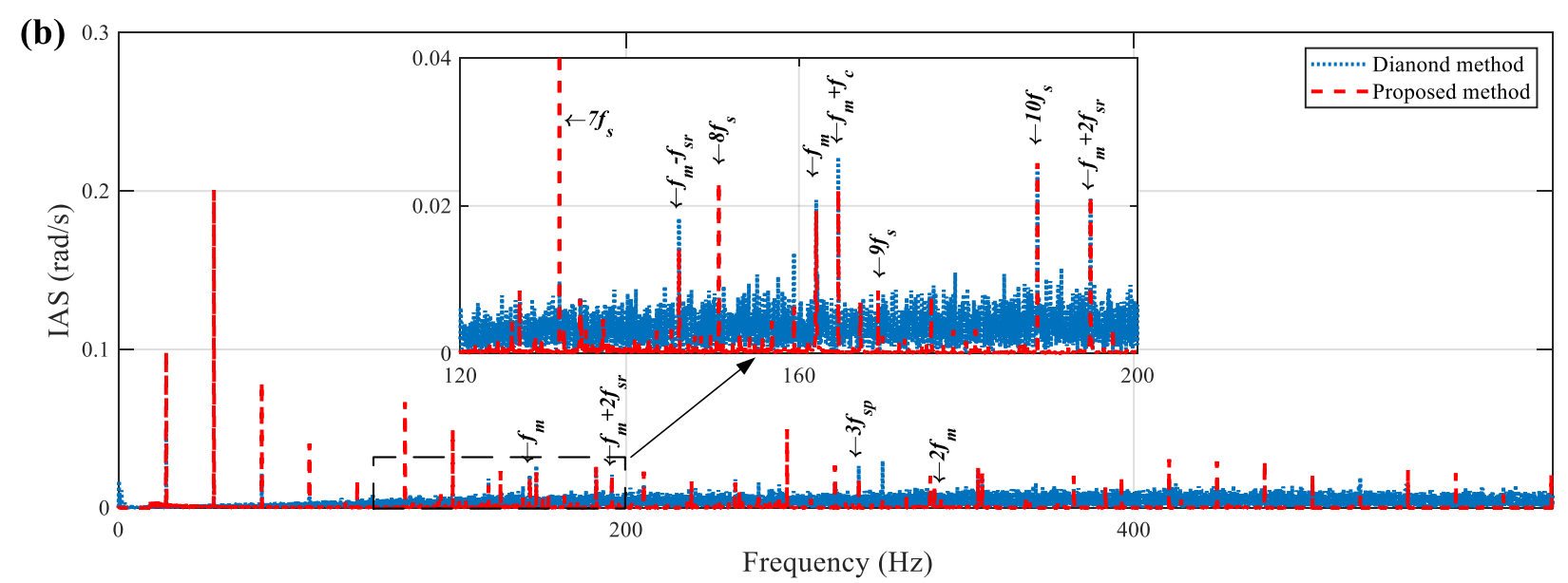

Fig. 13 IAS spectra (a) Resor method and proposed method (b) Diamond method and proposed method

Fig. 13 illustrates the frequency spectra of the three signals. Resor method eliminates all the harmonics of the rotating speed $f_{s}$, while these are kept in some degree by the Diamond and the proposed methods. Despite the angle interval corrected, we can still observe significant background noises in the processed IAS signals by Resor and Diamond methods due to the discrete time sampling. As can be seen from the red dash line in Fig. 13 (a) and (b), the proposed method suppresses the false IAS at integer $f_{s}$ harmonics, meanwhile, remains the non-integer IAS components. For example, mesh frequency $f_{m}$ and its sidebands like $f_{m}+f_{c}, f_{m}+f_{s r}$ and $f_{m}-f_{s r}$ can be clearly observed.

The IAS spectrum of the proposed method has a higher amplitude at the $9 f_{s}, 10 f_{s}, 11 f_{s}, 12 f_{s}$, as in the zoomed in subfigure. More harmonic amplitudes are listed in Fig. 14. The energy of the proposed method IAS spectrum is more concentrated at the $f_{s}$ harmonics, characteristic frequencies and their sidebands, rather than the whole frequency range. The amplitudes of the first 6 IAS harmonic of the proposed method are much higher, which is the opposite of the encoder error deviations, as in Fig. 10. It indicates that speed variation has been maintained rather than eliminated with encoder errors.

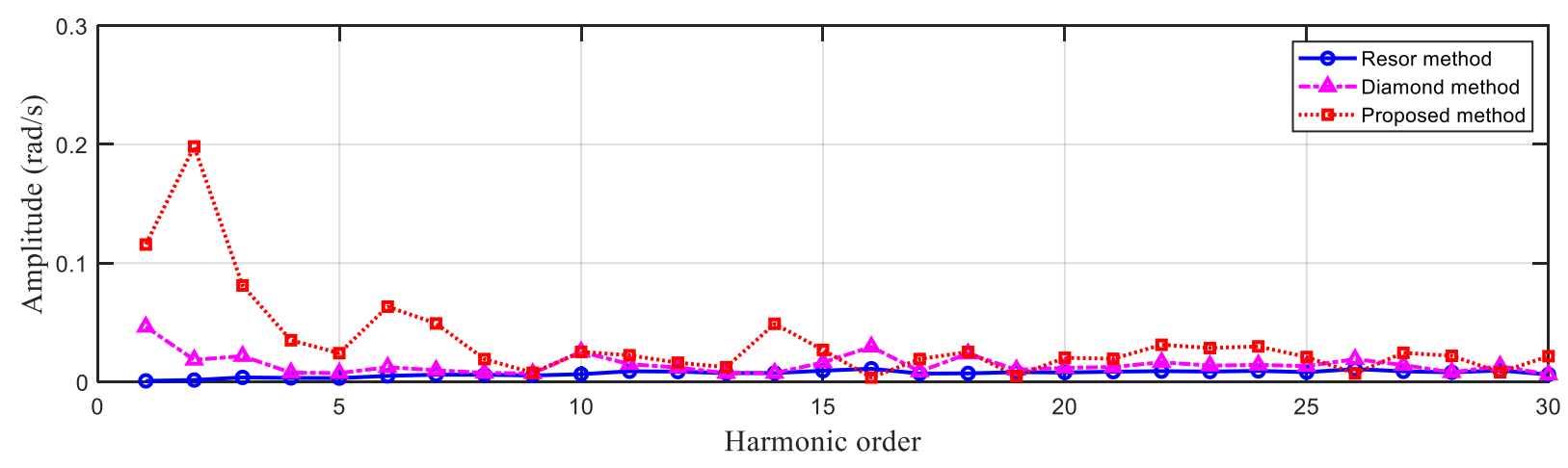

Fig. 14 IAS harmonics amplitude

From the IAS spectrums, in Fig. 13, it can be seen that the proposed method has a better SNR than the other methods in that plenty details such as the weak mesh harmonics and the sidebands are revealed. Besides, the encoder false IAS is also suppressed. The Resor method has no $f_{s}$ harmonic 
components, while the Diamond method remains shaft rotating speed variations at integer harmonics. The proposed method further improved the SNR by suppressing the false IAS components occurred at the rotating harmonics. To quantitatively analyse the superiority of the proposed method, the meanpeak ratios (MPR) of the characteristic harmonics component are compared with Resor and Diamond method. The definition of MPR can be found in [29][30]:

$$
M P R=20 \log _{10} \frac{\sum_{i=1}^{N}\left(p_{i}-A_{s}\right)}{A_{s}}
$$

where $A_{s}$ is the average value of the interested frequency range, $p_{i}$ is the peak value of the interested harmonics. $\mathrm{N}$ is the number of harmonics.

The value $A_{s}$ averaged from a $0-560 \mathrm{~Hz}$ frequency range, which covers the first 30 shaft rotating orders. The peak values of the first 10 interested harmonics of $f_{c}, f_{s}, f_{s r}, f_{p}, f_{p p}$, and first 3 harmonics of $f_{m}, f_{s p}$ are taken into consideration in MPR calculation.

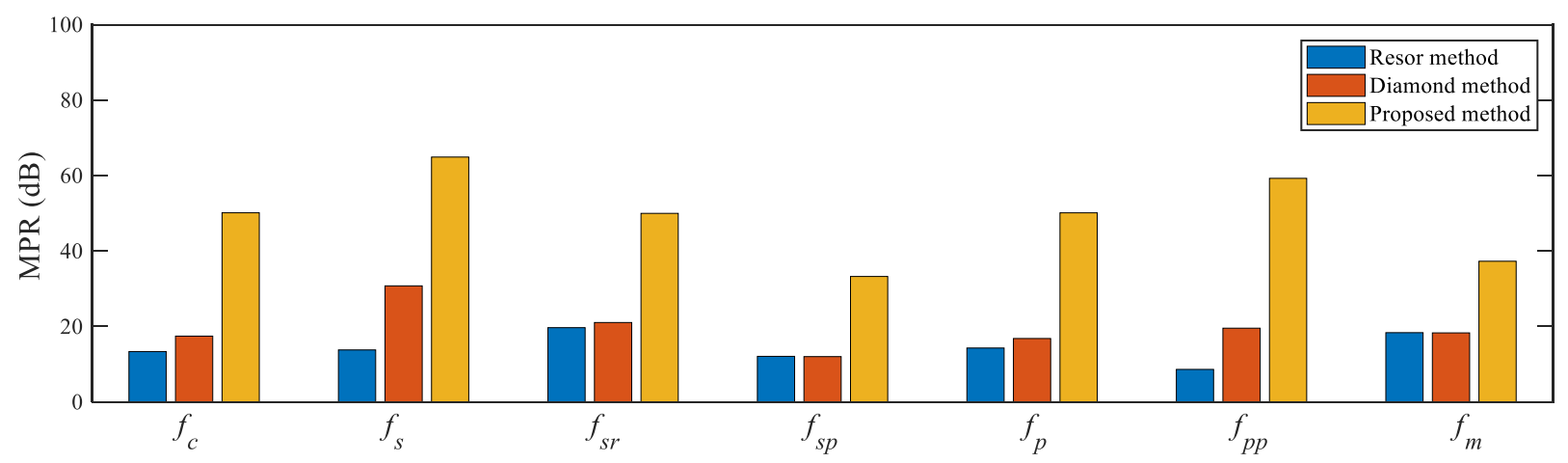

Fig. 15 MPR values of the three methods at different characteristic frequencies

The MPR values are shown in Fig. 15, the proposed method has a higher MPR value than other methods in all characteristic frequencies. The proposed method not only eliminate the encoder error false IAS but also has a much higher SNR, which reveals the weak IAS components from the background noise. As the PG is healthy, the high amplitude at the fault characteristic frequencies is a baseline and indicates the existed imperfection of the PG, rather than a fault.

\subsection{Discussion}

Based on the analysis of the experimental results, the following key observations are summarised.

1. Encoder error: The estimated encoder error by the Resor and the proposed method are similar. The proposed method acquires a comprehensive continuous encoder error from two different detector, while Resor method results in two slightly different encoder error profiles due to different measurement points and poor roughness of 3D printed encoder disc. The Resor method has larger amplitudes at the low orders in the encoder error spectrum (Fig. 10). It may be caused by that the Resor method mistakes the low-frequency true IAS fluctuation as encoder errors, and 
the slow changing lower order encoder error components are easier to suffer from the time quantification errors. It is also noticed that the proposed method avoids the encoder error missing at harmonics $R, 2 R, 3 R \ldots(R$ is the detector number), similar as other multiple detector encoder error compensation methods [18][20].

2. IAS waveform: The proposed method obtains a least deviated IAS waveform, as presented in Fig. 12. The test rig runs at a quasi-constant speed operation condition, therefore, it is reasonable to consider an IAS with smaller deviations be more accurate, as mentioned in [23]. The IAS regressed by the Diamond method has larger jitters at time stamps, which can be caused by the insufficient sampling rate. In our experiment, the rotating speed is two times larger than that in [23], while the sampling frequency are almost the same. Therefore, it causes the polynomialfitted IAS is no longer continuous at time stamps. To solve this problem, the fitted IAS is replaced by a pricewise averaged IAS, which has smaller jitters than fitted IAS but still larger than the proposed method. The proposed method acquires an IAS with the smallest standard deviation and the smallest kurtosis, as compared in Table 3. It indicates that the acquired IAS is smoother as in Fig. 12 (b). Therefore, the proposed dual detector method is a more accurate method to estimate the IAS signal.

3. IAS spectrum: The proposed method has much higher MPR values at all interested PG characteristic components compared to other methods. The false IAS at rotating harmonics are effectively suppressed, especial at higher orders. The energy in the IAS spectrum of the proposed method concentrates at the characteristic frequencies, which further improves the IAS signal SNR. In the IAS spectrums of the three improved methods, the first three rotating harmonics of the proposed method have significantly higher values than other methods. In the Resor and Diamond method, the estimation of the IAS fluctuation depends on the angle increment at each slot, while the low-frequency IAS fluctuation changes slowly and has a small angle increment at slots, which are more easily masked by the time quantification errors.

\section{Conclusions}

A novel method to accurately estimate IAS signal is developed based on a dual detector setup that allows false IAS components caused by encoder errors to be suppressed effectively. It is achieved by calculating the central difference of the IAS signals from the dual detectors along with a frequency domain integration. The accuracy of this technique are evaluated through a numerical study considering different influential factors. The dual detector interval angle acts like a low-pass filter, and a smaller interval angle gives a more accurate estimation of IAS. The average speed has negligible influences. The ratio of the interval angle to the encoder division angle is recommended to be less than $0.5^{\circ}$. Experimental results show that the encoder error estimated by the proposed method has higher amplitudes at lower orders of rotational speed. The IAS estimated by the proposed method has the smallest amplitudes of standard deviations and kurtosis but has the highest MPR at all interested 
frequency components. Therefore, it allows weak characteristic frequency components to be quantified more accurately, thus allowing rotor dynamics to be more correctly acquired. This is particularly significant for machine condition monitoring where minimising expenditure is an overriding factor.

\section{Reference}

[1] B. Li, X. Zhang, J. Wu, New procedure for gear fault detection and diagnosis using instantaneous angular speed, Mech. Syst. Signal Process. 85 (2017) 415-428. doi:10.1016/j.ymssp.2016.08.036.

[2] M. Zhao, X. Jia, J. Lin, Y. Lei, J. Lee, Instantaneous speed jitter detection via encoder signal and its application for the diagnosis of planetary gearbox, Mech. Syst. Signal Process. 98 (2018) 16-31. doi:10.1016/j.ymssp.2017.04.033.

[3] S. Xue, I. Howard, Torsional vibration signal analysis as a diagnostic tool for planetary gear fault detection, Mech. Syst. Signal Process. 100 (2018) 706-728. doi:10.1016/j.ymssp.2017.07.038.

[4] L. Renaudin, F. Bonnardot, O. Musy, J.B. Doray, D. Re, Natural roller bearing fault detection by angular measurement of true instantaneous angular speed, 24 (2011) 1998-2011. doi:10.1016/j.ymssp.2010.05.005.

[5] S.D. Yu, X. Zhang, A data processing method for determining instantaneous angular speed and acceleration of crankshaft in an aircraft engine-propeller system using a magnetic encoder, Mech. Syst. Signal Process. 24 (2010) 1032-1048. doi:10.1016/j.ymssp.2009.10.010.

[6] A.A. Gubran, J.K. Sinha, Shaft instantaneous angular speed for blade vibration in rotating machine, Mech. Syst. Signal Process. 44 (2014) 47-59. doi:10.1016/j.ymssp.2013.02.005.

[7] D. Abboud, J. Antoni, Order-frequency analysis of machine signals, Mech. Syst. Signal Process. 87 (2017) 229258. doi:10.1016/j.ymssp.2016.10.024.

[8] M.D. Coats, R.B. Randall, Single and multi-stage phase demodulation based order-tracking, Mech. Syst. Signal Process. 44 (2014) 86-117. doi:10.1016/j.ymssp.2013.09.016.

[9] F. Gu, I. Yesilyurt, Y. Li, G. Harris, A. Ball, An investigation of the effects of measurement noise in the use of instantaneous angular speed for machine diagnosis, Mech. Syst. Signal Process. 20 (2006) 1444-1460. doi:10.1016/j.ymssp.2005.02.001.

[10] C.L. Groover, M.W. Trethewey, K.P. Maynard, M.S. Lebold, Removal of order domain content in rotating equipment signals by double resampling, Mech. Syst. Signal Process. 19 (2005) 483-500. doi:10.1016/j.ymssp.2003.09.008.

[11] D. Rémond, J. Antoni, R.B. Randall, Instantaneous Angular Speed (IAS) processing and related angular applications, Mech. Syst. Signal Process. 45 (2014) 24-27. doi:10.1016/j.ymssp.2013.10.015.

[12] A. Rivola, M. Troncossi, Zebra tape identification for the instantaneous angular speed computation and angular resampling of motorbike valve train measurements, Mech. Syst. Signal Process. 44 (2014) 5-13. doi:10.1016/j.ymssp.2012.11.009.

[13] H. André, F. Girardin, A. Bourdon, J. Antoni, D. Rémond, Precision of the IAS monitoring system based on the elapsed time method in the spectral domain, Mech. Syst. Signal Process. 44 (2014) 14-30. doi:10.1016/j.ymssp.2013.06.020.

[14] S. Qin, Z. Huang, X. Wang, Optical angular encoder installation error measurement and calibration by ring laser gyroscope, IEEE Trans. Instrum. Meas. 59 (2010) 506-511. doi:10.1109/TIM.2009.2022104.

[15] M.N. Burnashev, P.A. Pavlov, Y. V Filatov, Development of precision laser goniometer systems Development of precision laser goniometer systems, (2013). doi:10.1070/QE2013v043n02ABEH015045.

[16] R.D. Geckeler, A. Fricke, C. Elster, Calibration of angle encoders using transfer functions, Meas. Sci. Technol. 17 (2006) 2811-2818. doi:10.1088/0957-0233/17/10/036.

[17] F. Deng, J. Chen, Y. Wang, K. Gong, Measurement and calibration method for an optical encoder based on adaptive differential evolution-Fourier neural networks, Meas. Sci. Technol. 24 (2013). doi:10.1088/0957- 
0233/24/5/055007.

[18] R. Probst, Self-calibration of divided circles on the basis of a prime factor algorithm, Meas. Sci. Technol. 19 (2008) 1-12. doi:10.1088/0957-0233/19/1/015101.

[19] Y. Jiao, Y. Ding, Z. Dong, M. Huang, P. Liu, Optimal-arrangement-based four-scanning- heads error separation technique for self- calibration of angle encoders, Meas. Sci. Technol. 29.8 (2018) 085005.

[20] T. Watanabe, H. Fujimoto, K. Nakayama, T. Masuda, M. Kajitani, Automatic high-precision calibration system for angle encoder, Proc. SPIE. 4401 (2001) 267-274. doi:10.1117/12.445630.

[21] B.R. Resor, M.W. Trethewey, K.P. Maynard, Compensation for encoder geometry and shaft speed variation in time interval torsional vibration measurement, J. Sound Vib. 286 (2005) 897-920. doi:10.1016/j.jsv.2004.10.044.

[22] H. André, F. Girardin, A. Bourdon, J. Antoni, D. Rémond, Precision of the IAS monitoring system based on the elapsed time method in the spectral domain, Mech. Syst. Signal Process. 44 (2014) 14-30. doi:10.1016/j.ymssp.2013.06.020.

[23] D.H. Diamond, P.S. Heyns, A.J. Oberholster, Online shaft encoder geometry compensation for arbitrary shaft speed profiles using Bayesian regression, Mech. Syst. Signal Process. 81 (2016) 402-418. doi:10.1016/j.ymssp.2016.02.060.

[24] Y. Li, F. Gu, G. Harris, A. Ball, N. Bennett, K. Travis, The measurement of instantaneous angular speed, Mech. Syst. Signal Process. 19 (2005) 786-805. doi:10.1016/j.ymssp.2004.04.003.

[25] K. Saito, K. Kamiyama, T. Ohmae, T. Matsuda, A Microprocessor-Controlled Speed Regulator With Instantaneous Speed Estimation For Motor Drives, IEEE Trans. Ind. Electron. 35 (1988) 95-99. doi:10.1109/41.3068.

[26] David Vakman, New high precision frequency measurement, Meas. Sci. Technol. 11 (2000) 1493-1497.

[27] Q. Zeng, I. Alqatawneh, Y. Xu, Y. Shao, F. Gu, A. Ball, The Improvement of Instantaneous Angular Speed Estimation using Signals from a Dual Read Head for Monitoring Planetary Gearboxes, Cond. Monit. Diagnostic Eng. Manag. (2018) 234.

[28] S. Braun, The synchronous ( time domain ) average revisited, Mech. Syst. Signal Process. 25 (2011) $1087-1102$. doi:10.1016/j.ymssp.2010.07.016.

[29] L. Wang, Y. Shao, Z. Cao, Optimal demodulation subband selection for sun gear crack fault diagnosis in planetary gearbox, Measurement. 125 (2018) 554-563. doi:10.1016/j.measurement.2018.05.023.

[30] A. Widodo, E.Y. Kim, J. Son, B. Yang, A.C.C. Tan, D. Gu, B. Choi, J. Mathew, Expert Systems with Applications Fault diagnosis of low speed bearing based on relevance vector machine and support vector machine, Expert Syst. Appl. 36 (2009) 7252-7261. doi:10.1016/j.eswa.2008.09.033. 